\title{
A Task Allocation Method Based on RMT of the Processor Core for MPSoC
}

\author{
Hui Ji a , Lei Zhou ${ }^{\text {b, * }}$ \\ College of Information Engineering, Yangzhou University, Yangzhong Jiangsu, China \\ a1053021992@qq.com; b tomcat800607@126.com
}

Keywords: MPSoCs, Task allocation, RMT, genetic algorithm.

\begin{abstract}
With the rapid development of integrated technology, more and more IP cores were integrated on a single chip. However, the improvement of the transistor density and processor working frequency resulted in increasing power density and heat generation. So MPSoCs (Multiprocessor System-on-Chips) are facing inevitable heat dissipation problems. In this paper, a task allocation method based on the regional mean temperature (RMT) of the processor core was proposesed. The method fully considers the regional temperature of processor cores by using vector distance to calculate temperature gradient and adopting genetic algorithm to assign the initial task. Experiment results indicate that, compared with the random task allocation strategy, the peak temperature reduction, hotspot reduction and temperature gradient reduction in RMT strategy can reach the maximum value of $4.69 \%, 42.31 \%$ and $77.49 \%$, respectively.
\end{abstract}

\section{Introduction}

With the development of integrated circuit technology, the increasing number of IP core can be integrated on the chip. Because of extensibility, delay, power dissipation and other factors, NoC (Network on Chip) has gradually become the mainstream way of communication between multicore. Traditional bus interconnection has been unable to adapt to the current communication system requirements of MPSoCs. Therefore, the newly-developing MPSoCs combined with the structure of NoC design has emerged. However, the improvement of transistor density and the working frequency make power consumption of the chip processor increased and result in MPSoCs processors in the overheating, which not only reduces the performance of the system, but also shortens the life of the device[1-3].Therefore, the heat distribution and temperature control is the key to designing MPSoCs.

However, system initial task allocation under the initial running state of system is extremely important for heat distribution and temperature control. At present, the research of heat-dissipation problem based on the software was classified into static task allocation and dynamic task scheduling. Static task allocation was divided into the offline algorithm and the online algorithm. The offline algorithm can use the external calculation to optimize the task allocation before the system is running. The online algorithm refers to the processor on chip computing tasks initial allocation methods when the system is running. However, because online task allocation algorithm allocating processor resources automatically according to the download tasks needs processors to consume system computing resources, therefore the complexity of the algorithm is restricted. Compared with the online algorithm, the offline algorithm can make full use of external computing resources to realize complex algorithm and obtain a better initial task allocation. Therefore, this paper chooses off-line algorithm to realize the initial task allocation scheme.

System's heat generation is mainly composed of processor power consumption and communication power consumption. When the system determines the tasks to be allocated, the processor power consumption has been determined. Communication power consumption is associated with the location of the task assigned. Even if the communication tasks have been determined, there exists great differences in communication power consumption under the different distribution. But this requirement and system heat distribution demand contradict each other: due to the fact that large traffic between tasks needs consume more processor power to obtain enough computing power. Therefore, from the point of the heat distribution, larger traffic between tasks had 
better to be allocated in the far apart area, in case the temperature rising results in higher power density and producing hot spots. This kind of allocation strategy causes the increase of communication power and risk of system overheating. Therefore, balanced communication power consumption and uniform heat distribution are of crucial importance. In [4], Zhu C proposed initial task allocation method according to off-line power equalization algorithm. This method uses HotSpot to simulation for task distribution and adjustment, which reduces the peak temperature of the chip and improves the system reliability. In literature[5], Han Wang put forward a thermal management strategy through temperature controller managing temperature to improve system performance. Lung C, in article[6], considering the undistributed task having influence on temperature distribution of global processor cores, put forward heat perception online task allocation strategy, which reduces task allocation complex and implement rapid task allocation. In [7], Cui and Zhang W Y presented an effective heat perception task scheduling algorithm called a Bottom-to-Top Scheme. Firstly, tasks are mapped to the bottom layer of 3D NoC, and then some tasks are selected and adjusted to the top for reducing the peak temperature and the execution time.

The existing allocation strategy only took the peak temperature into account without considering the difference temperature value of the processor core and its regional cores having an effect on the initial task assignment. On account of the above problems, this paper proposes a static task allocation method based on processor core regional mean temperature, which uses vector distance to calculate temperature gradient and employs genetic algorithm to complete the initial task optimal allocation. This method can reduce the peak temperature, the number of hotspot and the temperature gradient. The rest of the paper is organized as follows: Section II presents the regional mean temperature strategy(RMT), Section III illustrates the simulation results and Section IV concludes the paper.

\section{The RMT Strategy}

\subsection{The Influence of Regional Vector Distance}

In [8], according to the relationship among temperature, thermal resistance and power consumption, thermal model of IP core under steady state is as follows:

$$
T h_{i, j, k}=T_{\text {Amb }}+\sum_{m=1}^{k} \frac{R_{i, j, m}}{A} \times\left(\sum_{s=m}^{n} P_{i, j, s}+P R_{i, j, s}\right)
$$

In (1), $\mathrm{Th}_{i, j, k}$ shows the temperature of the PE at the position $(\mathrm{i}, \mathrm{j}, \mathrm{k})$ in $3 \mathrm{D}$ NoC. $\mathrm{T}_{\mathrm{Amb}}$ is the ambient temperature. $\mathrm{R}_{i, j, m}$ is the thermal resistance of the PE at the position (i,j, $\mathrm{m}$ ) in 3D NoC. The area of each $\mathrm{PE}$ is shown with $\mathrm{A} . \mathrm{P}_{i, j, s}$ is the average power consumption of the $\mathrm{PE}$ at the position $(\mathrm{i}, \mathrm{j}, \mathrm{s})$ in 3D NoC. PRi,j,s is the average power consumption of the router at the position $(\mathrm{i}, \mathrm{j}, \mathrm{k})$ in the 3D NoC. The formula shows that the power consumption of the IP core has the certain influence on the the temperature of processor cores. As shown in formula (2), $\mathrm{t}_{c i j}$ is the current temperature of $\mathrm{C}_{i j}$. $\mathrm{t}_{c i j}$ ' is the temperature of $\mathrm{C}_{i j}$ selected according to vector distance after power consumption change. $\sigma$ expresses growth rate of temperature for $\mathrm{C}_{i j}$. Figure 1 is $4 * 4$ mesh network and figure 2 is the corresponding vector diagram of figure 1. we choose Bpred_0 and select DTB_1, Bpred_2 and FPReg_1 as regional processor cores with the vector distance of 1,2, and 3, respectively. When power consumption of DTB_1 is increased by $30 \mathrm{w}$, Bpred_2(calculate $\sigma$ in formula (2) and FPReg_1 performs the same step. Bpred_0 temperature goes up by $20 \%, 5.631 \%, 4.360 \%$ and $4.135 \%$, respectively, as shown in figure 3, so there is a negative correlation between $\sigma$ and vector distance. This paper chooses the vector distance of 1 as regional processor cores to study the temperature difference of processor cores for achieving uniform heat distribution.

$$
\sigma=\frac{t_{c i j}^{\prime}-t_{c i j}}{t_{c i j}^{\prime}}
$$




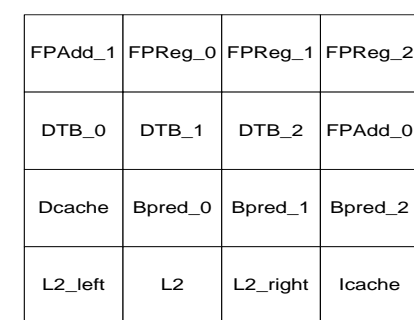

Fig $1.4 * 4$ mesh

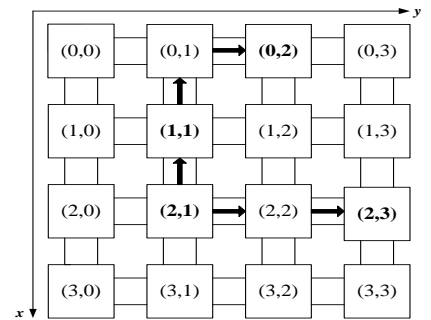

Fig 2 .the vector diagram of $4 * 4$ mesh

\subsection{The RMT Allocation Strategy}

In 2D MPSoC, considering the heat dissipation capacity between the layers and the temperature balance of processor cores having been allocated, it is quite complex for us to allocate processor cores to appropriate location. We assume $\mathrm{N}^{*} \mathrm{M}$ mesh. Each core can be expressed as $\mathrm{C}_{\mathrm{ij}}(1,2,3 \ldots \ldots, \mathrm{N}$ for $\mathrm{i} ; 1,2,3, \ldots, \mathrm{M}$ for $\mathrm{j})$ and adjacent nodes can be expressed as $\mathrm{A}_{(\mathrm{i}-1) \mathrm{j}}, \mathrm{A}_{(\mathrm{i}+1) \mathrm{j}}, \mathrm{A}_{\mathrm{i}(\mathrm{j}-1)}$ and $\mathrm{A}_{\mathrm{i}(\mathrm{j}+1)}$. According to the position of $C_{i j}$, the value of $A_{(i-1)}, A_{(i+1)}, A_{i(j-1)}$ and $A_{i(j+1)}$ can be set equal to 0 or 1 . For example, as shown in figure $4, \mathrm{~A}_{(\mathrm{i}-1) \mathrm{j}}, \mathrm{A}_{(\mathrm{i}+1) \mathrm{j}}, \mathrm{A}_{\mathrm{i}(\mathrm{j}-1)}$ and $\mathrm{A}_{\mathrm{i}(\mathrm{j}+1)}$ for FPAdd_1 are equal to $0,1,0$ and 1 and for DTB_1 are equal to $1,1,1$ and 1 . $\mathrm{T}_{\mathrm{Cij}}$ is temperature of $\mathrm{C}_{\mathrm{ij}}$ and $\mathrm{T}_{(\mathrm{i}-1) \mathrm{j}}, \mathrm{T}_{(\mathrm{i}+1) \mathrm{j}}, \mathrm{T}_{\mathrm{i}(\mathrm{j}-1)}$ and $\mathrm{T}_{\mathrm{i}(\mathrm{j}+1)}$ are the temperature of adjacent nodes.

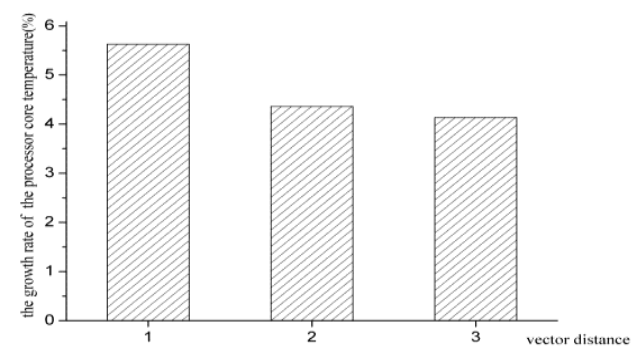

\begin{tabular}{|l|l|l|}
\hline & $A_{i(j-1)}$ & \\
\hline$A_{(i-1) j}$ & $C_{i j}$ & $A_{(i+1) j}$ \\
\hline & $A_{i(j+1)}$ & \\
\end{tabular}

Fig 3.the influence of regional vector distance Fig 4.the regional model of the processor core

Tavgis the average value of $\mathrm{T}_{(i-1) j}, \mathrm{~T}_{(i+1) j}, \mathrm{~T}_{i(j-1)}$ and $\mathrm{T}_{i(j+1)} . \Delta \mathrm{T}_{i j}$ is the difference of $\mathrm{T}_{C i j}$ and $\mathrm{T}_{a v g}$. $\Delta \mathrm{T}_{\text {avg }}$ is the average value of $\Delta \mathrm{T}_{i j}$. $\mu$ is the variance of $\Delta \mathrm{T}_{i j}$ as temperature gradient.

$$
\begin{aligned}
& T_{\text {avg }}=\frac{A_{(i-1) j} T_{(i-1) j}+A_{(i+1) j} T_{(i+1) j}+A_{i(j-1)} T_{i(j-1)}+A_{i(j+1)} T_{i(j+1)}}{A_{(i-1) j}+A_{(i+1) j}+A_{i(j-1)}+A_{i(j+1)}} \\
& \Delta T_{i j}=T_{C_{i j}}-T_{\text {avg }} \\
& \Delta T_{\text {avg }}=\frac{1}{N M} \sum_{i=1}^{N} \sum_{j=1}^{M} \Delta T_{i j} \\
& \mu=\frac{1}{N M} \sum_{i=1}^{N} \sum_{j=1}^{M}\left(\Delta T_{i j}-\Delta T_{\text {avg }}\right)^{2}
\end{aligned}
$$

Fitness decides chances of chromosome survival and is associated with the optimization goal of algorithm. In this paper, fitness function is defined as follows:

$$
f=\min \{\mu\}
$$

Mapping the IP cores onto the resource nodes in NoC architecture has belonged to a NP hard problem. Our objective is to use the genetic algorithm to optimize the temperature gradient within reasonable time. This method is as follows:

1. Generate an initial population of $\mathrm{n}$ chromosome using integer coding, which corresponds to the sequence number of tasks and whose length is equal to the number of tasks in a task graph. Each gene in the chromosome represents a task.

2. Use formula (7) to assess the fitness of each chromosome in population, then reserve minimum $\mu$ depositing in $\mu^{\prime}$.

3. Create a new population by applying three operators similar to natural selection operators selection, crossover and mutation. Crossover operation is shown in figure (5), according to the fitness selecting two parent individuals from the population (the bigger the fitness, the greater the chance of being selected) for crossover and put them in the population. Calculate fitness and select the minimum $\mu$ compared with $\mu^{\prime}$, then put minimum value into $\mu^{\prime}$. Then choose the optimal chromosome for mutation after crossover operation. As shown in figure (6), the maximum temperature processor 
cores(task 2) are compared with the lowest temperature processor core(task 9) to exchange position then put them in the population. Calculate fitness and select the minimum $\mu$ compared with $\mu$ ', then put optimal value into $\mu$ '.

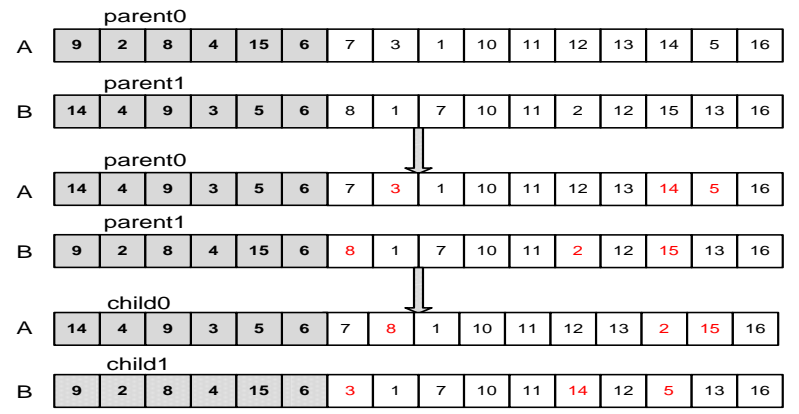

Fig 5.crossover operation

\begin{tabular}{|c|c|c|c|c|c|c|c|c|c|c|c|c|c|c|c|}
\hline 1 & 2 & 3 & 4 & 5 & 6 & 7 & 8 & 9 & 10 & 11 & 12 & 13 & 14 & 15 & 16 \\
\hline & II & & & & & & & II & & & & & & & \\
\hline 1 & 9 & 3 & 4 & 5 & 6 & 7 & 8 & 2 & 10 & 11 & 12 & 13 & 14 & 15 & 16 \\
\hline
\end{tabular}

Fig 6.mutation operation

4. Repeat the step 2 3 until temperature gradient has not changed for a sufficient number of iterations or number of iterations reach to maximum ,then stop and report the best mapping as the generated optimal IP core placement in the NoC.

In our implementation, we set the population size to be100, maximum generation to be 1500 , crossover probability to be 0.9 and mutation probability to be 0.01 . The length of the chromosome is equal to the number of IP cores.

\section{The Experimental Results Analysis}

\subsection{Experimental Environment}

In order to verify the validity of the RMT strategy, we choose $4 * 4$ mesh, $4 * 6$ mesh, $6 * 6$ mesh, $6 * 8$ mesh and $8 * 8$ mesh for this experiment. Task assignment of experiment is based on E3S [9] benchmark implement, which includes automation/industrial, office automation, networking, telecommunication and consumer-electronic. Each benchmark program consists of a task graph and a predetermined communication mode. Table 1 lists the detailed information of E3S benchmark.

Table 1 E3S Benchmark specification

\begin{tabular}{|c|c|c|}
\hline Benchmark category & Task graph & Number of tasks \\
\hline auto-indust & 4 & 24 \\
\hline consumer & 2 & 12 \\
\hline networking & 4 & 13 \\
\hline office-automation & 1 & 5 \\
\hline telecom & 9 & 30 \\
\hline
\end{tabular}

\subsection{The Result of the Experiment}

In order to guarantee the reliability of the running system, normally, peak temperature of processor cores should be between $85^{\circ} \mathrm{C} \sim 110^{\circ} \mathrm{C}$. In this article, the temperature of processing cores more than $85^{\circ} \mathrm{C}$ is called hotspots. The higher peak temperature of processor cores will lead to the decrease of reliability and validity for system. In the following, we compared the regional temperature distribution strategy (RMT) and random task allocation strategy (Random). We use the hotspot reduction, the peak temperature reduction and temperature gradient reduction to compare the performance of RMT solution and random task allocation solution. Hotspot reduction, peak temperature reduction and temperature gradient reduction can be represented with the following equations respectively.

\footnotetext{
Hotspot reduction $=\frac{\text { Hotspot }_{\mathrm{random}}-\text { Hotspot }_{\mathrm{RMT}}}{\text { Hotspot }_{\mathrm{random}}}$

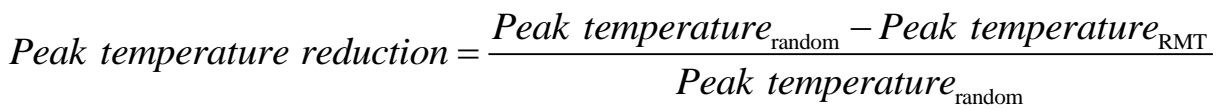




\section{Temperaure gradient reduction $=\frac{\mu_{\mathrm{random}}-\mu_{\mathrm{RMT}}}{\mu_{\mathrm{random}}}$}

Hotspot $_{\text {random }}$ is the number of hotspot for random task allocation. Hotspot RMT $_{\text {is }}$ is the number of

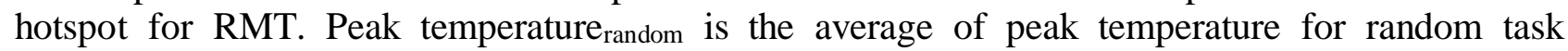
allocation. Peak temperature $\mathrm{RMT}_{\mathrm{T}}$ is peak temperature of RMT. $\mu_{\mathrm{RMT}}$ is the temperature gradient of RMT. $\mu_{\text {Random }}$ is the temperature gradient of random task allocation.

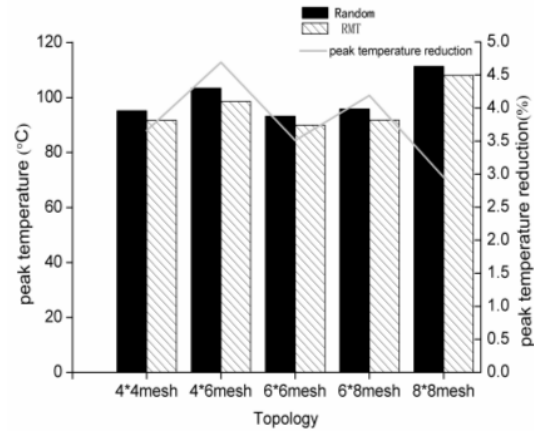

Fig 7. Peak temperature

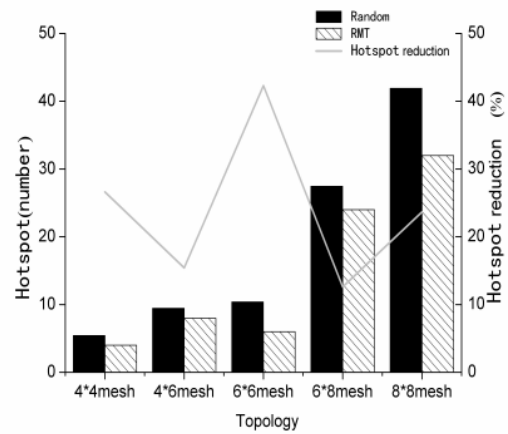

Fig 8.hotspot

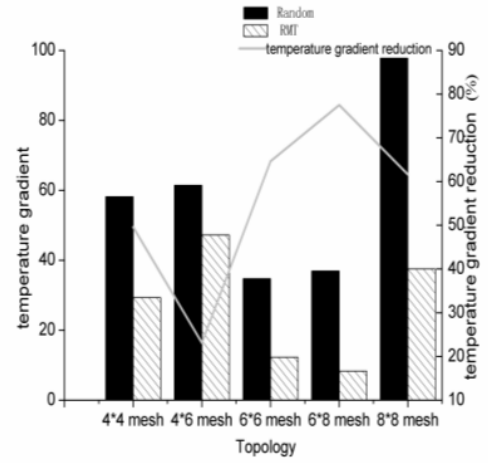

Fig 9.temperaure gradient

As shown in figure 7, figure 8 and figure 9 , the experiment selects $4 * 4$ mesh, $4 * 6$ mesh, $6 * 6$ mesh, 6*8 mesh and $8 * 8$ mesh networks, and then we compare two kinds of task allocation strategy: peak temperature reduction is $3.66 \%, 4.69 \%, 3.51 \%, 4.19 \%$ and $2.95 \%$. Hotspot reduction is $26.61 \%$, $15.43 \%, 42.31 \%, 12.6 \%$ and $23.66 \%$, respectively. Temperature gradient reduction is $49.59 \%$, $23.13 \%, 64.63 \%, 77.49 \%$ and $61.63 \%$. It's obvious, through the distribution of RMT, that peak temperature, the number of hotspot and temperature gradient are significantly lower, which makes the global heat distribution more uniform and improves reliability and validity of system.

\section{Conclusion}

For the heat dissipation problem in MPSoC, in this paper, we propose a regional mean temperature (RMT) method of the processor core. This method fully considers the regional temperature of processor cores by adopting genetic algorithm to assign the initial task. Compared with the random task allocation strategy, RMT reduces the number of hotspot, lower peak temperature and decrease the temperature gradient of the chip, which improve system's reliability and validity. In the future, we will focus on real-time temperature sensing aspects and for further studies.

\section{Acknowledgements}

This work is supported partly by National Natural Science Foundation of China under Grant No.61376025 and No.61301111, Natural science fund for colleges and universities in Jiangsu Province under Grant No.13KJB510039, Natural science Youth fund in Yangzhou city under Grant SQN20150035.

\section{References}

[1]. Xiang D, Chakrabarty K, Fujiwara H. Multicast-Based Testing and Thermal-Aware Test Scheduling for 3D ICs with a Stacked Network-on-Chip [J]. IEEE Transactions on Computers, 2016, 65(9):2767-2779.

[2]. Bahrebar P, Stroobandt D. Adaptive routing in MPSoCs using an efficient path-based method[C]// Soc Design Conference. IEEE, 2013:031-034.

[3]. Wei L, Zhou L. An equilibrium partitioning method for multicast traffic in 3D NoC architecture[C]//Very Large Scale Integration (VLSI-SoC), 2015 IFIP/IEEE International Conference on. IEEE, 2015: 128-133. 
[4]. Zhu C, Gu Z, Shang L, et al. Three-Dimensional Chip-Multiprocessor Run-Time Thermal anagement[J]. IEEE Transactions on Computer-Aided Design of Integrated Circuits and Systems, 2008, 27(8):1479-1492.

[5]. Wang H, Fu Y, Liu T, et al. Thermal management via task scheduling for 3D NoC based multiprocessor[C]//SoC Design Conference (ISOCC), 2010 International. IEEE, 2010: 440-444.

[6]. Lung C L, Ho Y L, Kwai D M, et al. Thermal-aware on-line task allocation for 3D multi-core processor throughput optimization[C]// Design, Automation \& Test in Europe Conference \& Exhibition (DATE), 2011. IEEE, 2011:1-6.

[7]. Cui Y, Zhang W, Chaturvedi V, et al. Thermal-Aware Task Scheduling for 3D-Network-on-Chip: A Bottom to Top Scheme [J]. Journal of Circuits, Systems and Computers, 2016, 25(01): 1640003.

[8]. Zhu C, Gu Z, Shang L, et al. Three-Dimensional Chip-Multiprocessor Run-Time Thermal Management [J]. IEEE Transactions on Computer-Aided Design of Integrated Circuits and Systems, 2008, 27(8):1479-1492.

[9]. Ghosh P, Sen A, Hall A. Energy efficient application mapping to NoC processing elements operating at multiple voltage levels[C]// International Symposium on Networks-On-Chips, NOCS 2009, May 10-13 2009, La Jolla, Ca, Usa. Proceedings. DBLP, 2009:80-85. 